\title{
Peertechz
}

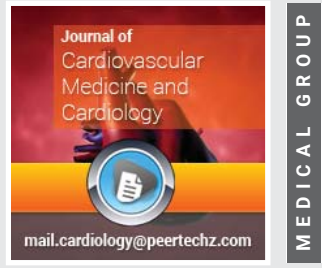

\section{An Atypical Case of Extrapulmonary Presentation of SARS-Cov-2 Infection}

\author{
Thet Tun ${ }^{1 *}$ and Merhej Hannun ${ }^{2}$ \\ ${ }^{1}$ Senior Clinical Fellow, Department of Cardiology, Bedford Hospital, UK \\ ${ }^{2}$ Foundation Year One, Department of Cardiology, Bedford Hospital, UK
}

Received: 23 November, 2020

Accepted: 03 December, 2020

Published: 04 December, 2020

*Corresponding author: Dr. Thet Tun, Senior Clinical Fellow, Department of Cardiology, Bedford Hospital, UK, Tel: +447833383394; E-mail: Thet.Tun@bedfordhospital.nhs.uk, thet.tunann@live.com

https://www.peertechz.com

Check for updates

\begin{abstract}
We present a case of a 26-year-old male who presented with diarrhoea, vomiting, chest pain and fever without respiratory symptoms including interstitial pneumonitis/ pneumonia. He deteriorated with hypotension, persistently high fever and multiorgan involvement. Troponins were elevated and Electrocardiogram (ECG) showed nonspecific T-wave and ST changes. Initial echocardiography showed severely reduced ejection fraction. He tested positive for a PCR nasopharyngeal swab for SARS-CoV-2. The patient recovered with about 10 days of only supportive care and his cardiac function returned to baseline, even though initial echocardiography had shown severely reduced left ventricular function. Although COVID-19 manifests primarily as a respiratory disease, few cases of cardiac injury without respiratory involvement have been reported. This case illustrates that COVID-19 can present atypically and affect multiorgan systems without significant respiratory system involvement.
\end{abstract}

\section{Introduction}

SARS-CoV-2 is a single-strand RNA virus from the Coronaviridae family; it can affect various organs including, but not limited to, the respiratory, renal, gastrointestinal, and cardiovascular systems. Myocarditis, or inflammation of the heart muscle, is a condition caused by the body's immune response to infection. Viral infections, generally, are one of the most common causes of heart muscle inflammation. Among viruses, the most cited are enteroviruses (especially coxsackievirus), adenovirus, parvovirus $\mathrm{B} 19$, and rarely coronavirus [1].

Coronavirus disease 2019 (COVID-19) was first described in Wuhan, China, in patients complaining of flu-likesymptoms. The virus was isolated and identified as a new strain of coronavirus, now named SARS-CoV-2 (severe acute respiratory syndrome coronavirus 2). SARS-CoV-2 not only causes viral pneumonia but has major implications for the cardiovascular system. Patients with cardiovascular risk factors including male sex, advanced age, diabetes, hypertension and obesity as well as patients with established cardiovascular and cerebrovascular diseases have been identified as particularly vulnerable populations with increased morbidity and mortality when suffering from COVID-19. Moreover, a considerable proportion of patients may develop cardiac injury in the context of COVID-19 which portends an increased risk of in-hospital mortality [2].

Although the pathophysiology of acute myocarditis of SARS-CoV-2 is not fully understood, several mechanisms have been proposed. The possible pathophysiology is that COVID-19related myocarditis may be caused by a combination of direct viral injury and the host's own auto-immune response. The virus attacks the body by attaching to angiotensin-convertingenzyme-2 (ACE2) receptors which are found in lung cells and other organs such as the heart and vessels.

A second probable mechanism is the indirect injury of the myocardium due to the cytokine storm that the infection triggers, demonstrated by the increase of interleukins: IL-6, $\mathrm{IL}-10, \mathrm{IL}-2$ receptor (IL-2R), and tumour necrosis factor alpha (TNF) $-\alpha$ in severe cases leading to the possibility of significant cellular damage.

\section{Case report}

A healthy and physically active 26-year-old Caribbean man with no significant past medical history presented to the accident and emergency department on $26^{\text {th }}$ October, 2020 with a chief complain of green diarrhoea and vomiting for 1 
week. He also had a fever of around $38.5^{\circ} \mathrm{C}$ and chills 3 days prior to admission and noted a rash on his torso and upper arms for one day. He had poor appetite, weakness, headache, generalized aches and abdominal pain. He was initially seen by a GP who thought it was a viral illness and discharged him with a prescription for analgesia. On the day of admission, he also experienced stabbing left sided chest pain of $8 / 10$ severity, which was worse with leaning forward but not exacerbated by movements or deep breathing [3] (Figure 1).

A week prior to the onset of symptoms, the patient ate out with his girlfriend at a restaurant and had a history of contact with a covid-19 positive patient a month before that. He is a non-smoker and all his family members including his girlfriend were symptom free. He also had no recent travel history and had not been out in nature recently.

On examination, the patient was clinically unwell and dehydrated. Observations showed heart rates of 113-120, systolic blood pressures of 92-96, a respiratory rate of 20 and oxygen saturations of $100 \%$. A blanching confluent erythematous macular rash on his upper chest and upper arms was noted. His chest was clear to auscultation and his abdomen was soft, but with generalized tenderness and no guarding. Heart sounds were normal to auscultation [4] (Figures 2,3).

\section{Initial investigations showed}

CRP: $335 \mathrm{mg} / \mathrm{L}$, WCC: 23.5 , sodium: $129 \mathrm{mmol} / \mathrm{L}$, potassium: 4.3, mmol/L, creatine: 177 umol/L, urea: $7.6 \mathrm{mmol} / \mathrm{l}$, troponin

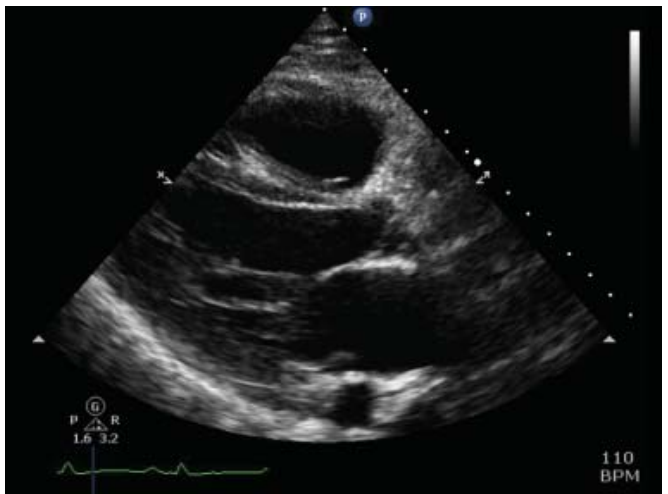

Figure 1: Transthoracic Echocardiography (long axis view).

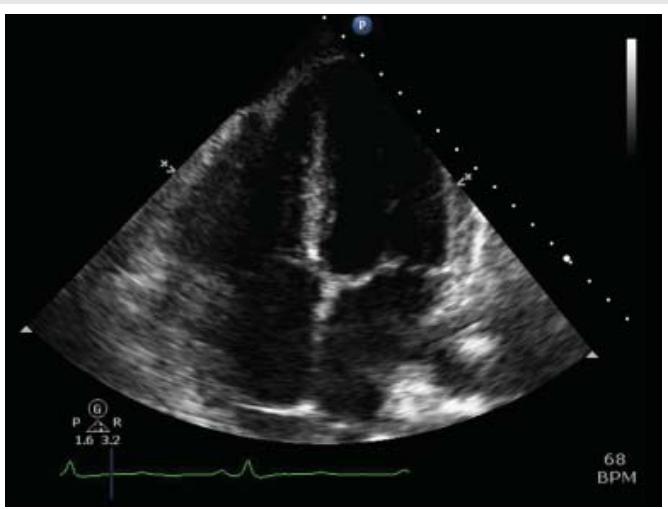

Figure 2: Transthoracic Echocardiography (Four Chamber View).

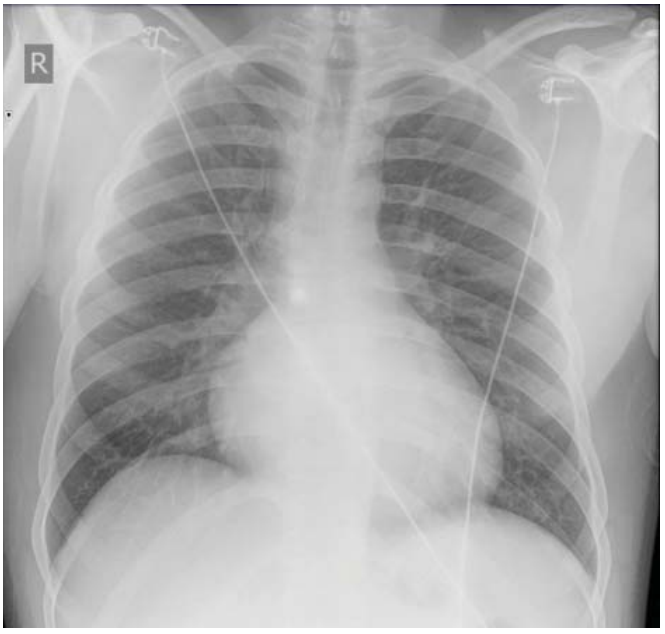

Figure 3: Normal Chest X-ray.

T: 457 ng/L, d-dimer: 977ng/ml, INR: 1.4 and prothrombin time: 16.4 .

He initially tested positive for SARS-CoV-2, but two subsequent tests were negative one week later. His Covid antibody test was positive for both IgG and IgM.

Electrocardiogram (ECG) revealed sinus tachycardia with partial RBBB, mild ST-depression in leads V3-V5 and T-wave inversion in the inferior leads. An initial chest radiograph demonstrated a normal cardiothymic silhouette with no focal consolidation, pleural effusion, or pneumothorax [5] (Figure 4).

CT pulmonary angiogram was done in view of high D-Dimers and pleuritic chest pain. No pulmonary embolism was found, however there was a small pleural effusion in the right lung measuring $2 \mathrm{~cm}$ in depth. There was also a small trace of pleural fluid in the left one. The lungs were otherwise clear with no significant mediastinal or hilar lymphadenopathy (Table 1).

He was Initially treated with intravenous fluids and antibiotics due to recurrent spiking fevers and dehydration at first presentation. Initial transthoracic echocardiogram demonstrated severely reduced left ventricular ejection fraction without obvious intracardiac clots or pericardial effusion. He also had severely impaired right ventricular systolic function, a borderline dilated right ventricle, severe tricuspid regurgitation, a PASP of $35 \mathrm{mmHg}+\mathrm{RAP}>15 \mathrm{mmHg}$, and moderate mitral regurgitation [6].

The patient's condition had not improved as he was regularly spiking fevers and remained hypotensive during his admission. In view of his low blood pressure, ongoing fevers and multiple organ involvement, several consultations were made with the intensive care, heart failure, and infectious disease specialists.

As per the instructions of the infectious disease consultant from Addenbrooke's Hospital, we treated the patient with intravenous ceftriaxone and metronidazole for 10 days in view 


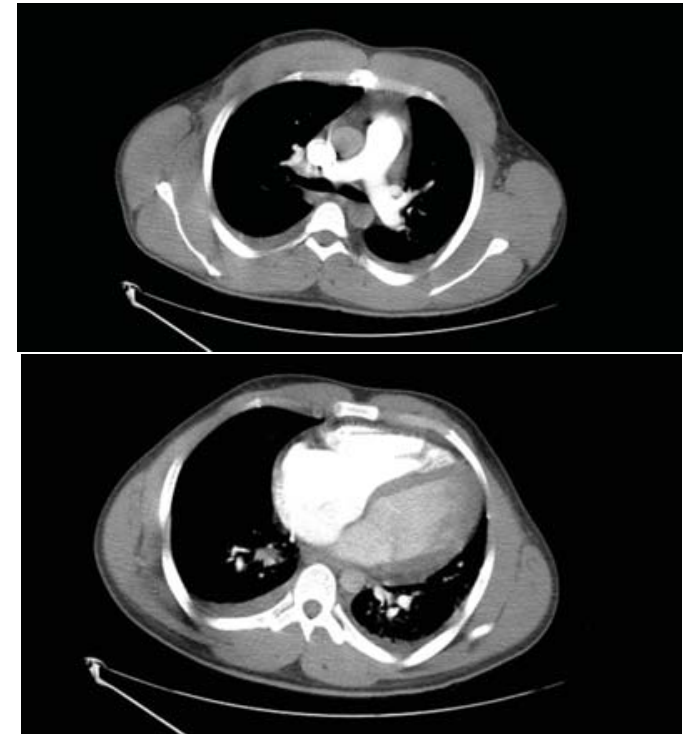

Figure 4: CT Pulmonary Angiogram: No Pulmonary Embolism, Small Pleural Effusion in the Right Lung $\sim 2 \mathrm{~cm}$ in depth and a Small Trace of Pleural Fluid in the Left One.

Table 1: A Snapshot Of the Patient's Blood Test Results.

\begin{tabular}{|c|c|c|c|c|c|}
\hline \multicolumn{6}{|c|}{ C-reactive protein } \\
\hline C-Reactive Protein & $(0-4.9)$ & $\mathrm{mg} / \mathrm{L}$ & & & *290.9 \\
\hline C-Reactive Protein & & & & & \\
\hline \multicolumn{6}{|c|}{ Double stranded dna antibody elisa } \\
\hline $\begin{array}{l}\text { Double Stranded DNA Antibody } \\
\text { (ELISA) }\end{array}$ & $(0-99)$ & $\mathrm{IU} / \mathrm{ML}$ & & 1.0 & \\
\hline \multicolumn{6}{|c|}{ Full blood count } \\
\hline Basophils & $(0.02-0.1)$ & $10^{9} / \mathrm{L}$ & 0.04 & & 0.08 \\
\hline Eosinophils & $(0-0.4)$ & $10^{9} / \mathrm{L}$ & $\star 0.50$ & & 0.34 \\
\hline Haematocrit & $(0.41-0.51)$ & L/L & $\star 0.33$ & & $* 0.33$ \\
\hline Haemoglobin & $(130-165)$ & $g / L$ & *11 & & *109 \\
\hline Lymphocytes & $(1-3)$ & $10^{9} / \mathrm{L}$ & ${ }^{*} 0.62$ & & 1.12 \\
\hline $\mathrm{MCHC}$ & $(280-355)$ & $g / L$ & 342 & & 333 \\
\hline MCV & $(80-100)$ & $\mathrm{fl}$ & 89 & & 89 \\
\hline Mean cell haemoglobin & $(27-32)$ & $\mathrm{pg}$ & 30 & & 30 \\
\hline Monocytes & $(0.2-1)$ & $10^{9} / \mathrm{L}$ & 0.32 & & 0.7 \\
\hline MPV & $(7.8-11)$ & $\mathrm{fL}$ & 10.2 & & 10.6 \\
\hline Neutrophils & $(2-7)$ & $10^{9} / \mathrm{L}$ & *18.43 & & *25.85 \\
\hline NRBC & $(0-0.5)$ & $10^{9} / \mathrm{L}$ & & & $<0.5$ \\
\hline Platelets & $(150-450)$ & $10^{9} / \mathrm{L}$ & *140 & & 215 \\
\hline $\mathrm{RBC}$ & $(4.5-5.9)$ & $10^{12} / \mathrm{L}$ & $\star 3.8$ & & *3.7 \\
\hline RDW & $(11.8-14.8)$ & $\%$ & 13.9 & & 14 \\
\hline White Cell Count & $(4-11)$ & $10^{9} / \mathrm{L}$ & *19.9 & & *28.1 \\
\hline \multicolumn{6}{|c|}{ Glucose (random - serum) } \\
\hline Glucose (Random - Serum) & & $\mathrm{mmol} / \mathrm{L}$ & 5.7 & & \\
\hline \multicolumn{6}{|c|}{ Lactate dehyrogenase (Idh) } \\
\hline Lactate Dehydrogenase (LDH) & $(0-249)$ & $\mathrm{U} / \mathrm{L}$ & & & *333 \\
\hline \multicolumn{6}{|c|}{ Liver function test } \\
\hline Alanine Aminotransferase (ALT) & $(0-40)$ & $\mathrm{U} / \mathrm{L}$ & *41 & & *92 \\
\hline Calculated Globin & & $g / L$ & 30 & & 35 \\
\hline Total Bilirubin & $(0-20)$ & umol/L & *26 & & $\star 24$ \\
\hline Total Protein & $(60-80)$ & $g / L$ & *50 & & *55 \\
\hline
\end{tabular}

of the persistent fever with abdominal pain. He did not need dexamethasone as there was no pulmonary involvement (Table 2).

Generally, glucocorticoid and immunoglobulin therapy are discouraged in acute myocarditis. Moreover, a systematic review of IV immunoglobulins as acute myocarditis therapy showed insufficient evidence to support their routine use.

In an effort to search for other common aetiologies of myocarditis, multiple respiratory and gastrointestinal pathogen investigations were done and found to be negative, such as: malaria, legionella and pneumococcal urinary antigen, urine and blood cultures, faecal studies for cryptosporidium, salmonella, shigella, campylobacter, E.coli, C.difficle, a throat swab culture , HIV, viral hepatitis screening, infectious mononucleosis, enterovirus, leptospirosis , EBV (negative IgM but positive IgG), influenza and parvovirus. Anti-streptolysin $\mathrm{O}$ titer was also very low and less than $200 \mathrm{IU} / \mathrm{ml}$. Vasculitis and autoimmune screening tests were also negative [7] (Figure 5).

Table 2: Increased Troponin T Results.

\begin{tabular}{|c|c|c|c|c|}
\hline \multicolumn{5}{|c|}{ Renal function } \\
\hline Creatinine (Jaffe) & $(62-106)$ & umol/L & *147 & \\
\hline 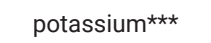 & $(3.5-5.3)$ & $\mathrm{mmol} / \mathrm{L}$ & 3.7 & \\
\hline Sodium & $(133-146)$ & $\mathrm{mmol} / \mathrm{L}$ & 133 & \\
\hline \multicolumn{5}{|c|}{ Troponin $\mathrm{t}$} \\
\hline Cardiac troponin $\mathrm{T}$ & $(0-14)$ & $\mathrm{ng} / \mathrm{L}$ & *342.4 & *314.9 \\
\hline
\end{tabular}

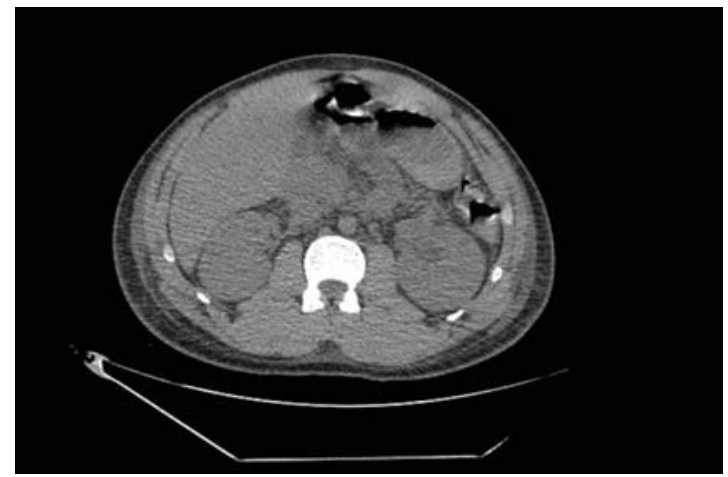

Figure 5: CT Abdomen and Pelvis in View of Deranged LFTs: No Organomegaly, Focal Lesions, Hydronephrosis, or Free Air Noted.

CT Abdomen and Pelvis was also done due to severe abdominal pain and deranged liver function tests. The liver, gallbladder, CBD, pancreas, spleen, both adrenal glands and kidneys were within normal limits. No focal lesions, hydronephrosis, or free air was noted (Table 3).

His inflammatory markers and clinical picture started to slowly improve after a week's time of conservative treatment in the hospital. His CRP fell from around 300 to $6.2 \mathrm{mg} / \mathrm{L}$.

His renal function was monitored closely, with initial creatinine found to be $177 \mathrm{umol} / \mathrm{L}$ which dropped to $82 \mathrm{umol} / \mathrm{L}$ at the time of discharge (Table 4). 
Moreover, his deranged LFT's also normalized. A repeat transthoracic echocardiography on November $5^{\text {th }}, 2020$ found

Table 3: Creatinine Trend Showing Initial AKI.

\begin{tabular}{|c|c|c|}
\hline 25 Oct 2020 20: $46: 00$ & 20B50170565 & *177 \\
\hline 26 Oct 2020 06:56:00 & 20B50170624 & *147 \\
\hline 27 Oct 2020 15:00:00 & 20B50198333 & *157 \\
\hline 28 Oct 2020 05:00:00 & 20B50170691 & *135 \\
\hline 28 Oct 2020 11:30:00 & 20B50197211 & *132 \\
\hline 28 Oct 2020 11:30:00 & 20B50197212 & *139 \\
\hline 29 Oct 2020 11:30:00 & 20B50197559 & 105 \\
\hline 30 Oct 2020 13:10:00 & 20B50199886 & 81 \\
\hline 31 Oct 2020 10:25:00 & 20B50200095 & 88 \\
\hline 01 Nov 2020 10:31:00 & 20B50200337 & 78 \\
\hline 02 Nov 2020 10:00:00 & 20B50203328 & 76 \\
\hline 03 Nov 2020 11:10:00 & 20B50203192 & 71 \\
\hline 04 Nov 2020 11:50:00 & 20B50201811 & 69 \\
\hline
\end{tabular}

his left ventricular systolic function to be normal with a visual left ventricular ejection fraction of 55-60\%, normal diastolic function, normal right ventricular size and function, normal atria, mild TR and PR, and a small pericardial effusion.

Around hospital day number 7, his blood pressure normalized and the patient became afebrile with a heart rate ranging from 80-100 bpm. His oxygen saturation on room air was $>95 \%$ throughout the rest of his stay [8].

Though tinzaparin was started on admission at a prophylactic dose due to concerns of elevated risk of clotting among patients with COVID-19, it had to be stopped due to coagulopathy. While attempts were made early to obtain antiviral treatment, the infectious disease team decided to hold this treatment given his overall improvement with supportive care. Dexamethasone, other anti-inflammatory agents and IVIG were deemed indicated. Medical treatment of his heart failure included furosemide, angiotensin-converting enzyme inhibitors and beta-blockers (Table 5).

Table 4: Trend of Deranged Blood Results [1].

\begin{tabular}{|c|c|c|c|c|c|c|c|c|}
\hline \multicolumn{9}{|c|}{ C-reactive protein } \\
\hline C-Reactive Protein & $(0-4.9)$ & $\mathrm{mg} / \mathrm{L}$ & $\star 57.5$ & *32.3 & *19.8 & *13.9 & $\star 8.8$ & *6.2 \\
\hline \multicolumn{9}{|c|}{ Full blood count } \\
\hline Basophils & $(0.02-0.1)$ & $10^{9} / \mathrm{L}$ & *0.12 & 0.08 & $\star 0.11$ & $\star^{*} 0.16$ & 0.1 & \\
\hline Eosinophils & $(0-0.4)$ & $10^{9} / \mathrm{L}$ & *0.52 & 0.4 & 0.36 & 0.38 & 0.27 & \\
\hline Haematicrit & $(0.41-0.51)$ & $L / L$ & $\star 0.34$ & $\star 0.33$ & $\star 0.32$ & *0.35 & $\star 0.37$ & \\
\hline Haemoglobin & $(130-165)$ & $g / L$ & *111 & *109 & *108 & *114 & *125 & \\
\hline Lymphocytes & $(1-3)$ & $10^{9} / \mathrm{L}$ & 1.4 & 1.43 & 1.53 & 1.58 & 2.37 & \\
\hline $\mathrm{MCHC}$ & $(280-355)$ & $g / L$ & 330 & 334 & 335 & 328 & 324 & \\
\hline MCV & $(80-100)$ & $\mathrm{fl}$ & 89 & 90 & 90 & 91 & 91 & \\
\hline Mean cell haemoglobin $(\mathrm{MCH})$ & $(27-32)$ & $\mathrm{pg}$ & 30 & 30 & 30 & 30 & 31 & \\
\hline Monocytes & $(0.2-1)$ & $10^{9} / \mathrm{L}$ & 0.86 & 0.96 & 0.99 & *1.01 & *1.11 & \\
\hline MPV & $(7.8-11)$ & $\mathrm{fL}$ & 8.3 & 8.4 & 8.4 & 7.9 & *7.4 & \\
\hline Neutrophils & $(2-7)$ & $10^{9} / \mathrm{L}$ & *16.30 & *12.30 & *9.51 & *10.56 & 6.75 & \\
\hline NRBC & $(0-0.5)$ & $10^{9} / \mathrm{L}$ & $<0.5$ & & & $<0.5$ & $<0.5$ & \\
\hline Platelets & $(150-450)$ & $10^{9} / \mathrm{L}$ & *650 & $\star 759$ & $\star 848$ & *976 & *1159 & \\
\hline RBC & $(4.5-5.9)$ & $10^{12} / \mathrm{L}$ & *3.8 & *3.6 & *3.6 & *3.8 & *4.1 & \\
\hline RDW & $(11.8-14.8)$ & $\%$ & 14.5 & *15.2 & 14.6 & *15.5 & *15.5 & \\
\hline \multicolumn{9}{|l|}{ White Cell Count } \\
\hline White Cell Count & $(4-11)$ & $10^{9} / \mathrm{L}$ & *19.2 & *15.5 & *12.5 & *13.7 & 10.6 & \\
\hline \multicolumn{9}{|c|}{ Liver function test } \\
\hline Alanine Aminotransferase (ALT) & $(0-40)$ & $U / L$ & $\star 53$ & & $\star 51$ & & *60 & $\star 54$ \\
\hline Calculated Globin & & $g / L$ & 41 & & 47 & & 57 & 51 \\
\hline Total Bilrubin & $(0-20)$ & umol/L & 11 & & 9 & & 12 & 11 \\
\hline Total Protein & $(60-80)$ & $g / L$ & $\star 59$ & & 69 & & $\star 88$ & 80 \\
\hline \multicolumn{9}{|c|}{ Magnesium } \\
\hline Magnesium & $(0.7-1)$ & $\mathrm{mmol} / \mathrm{L}$ & 0.78 & & 0.79 & & 0.85 & 0.8 \\
\hline \multicolumn{9}{|c|}{ Renal function } \\
\hline Creatinline (Jaffe) & $(62-106)$ & umol/L & 76 & 71 & 69 & 70 & 89 & 82 \\
\hline Potassium & $(3.5-5.3)$ & $\mathrm{mmol} / \mathrm{L}$ & 3.7 & 4.5 & 5.2 & 5 & $\star 5.4$ & 5 \\
\hline Sodium & $(133-146)$ & $\mathrm{mmol} / \mathrm{L}$ & 135 & 133 & 133 & 133 & 135 & 133 \\
\hline & & & & & & & & 299 \\
\hline
\end{tabular}

Citation: Tun T, Hannun M (2020) An Atypical Case of Extrapulmonary Presentation of SARS-Cov-2 Infection. J Cardiovasc Med Cardiol 7(4): $296-301$. 
Table 5: Trend of Deranged Blood Results [2]

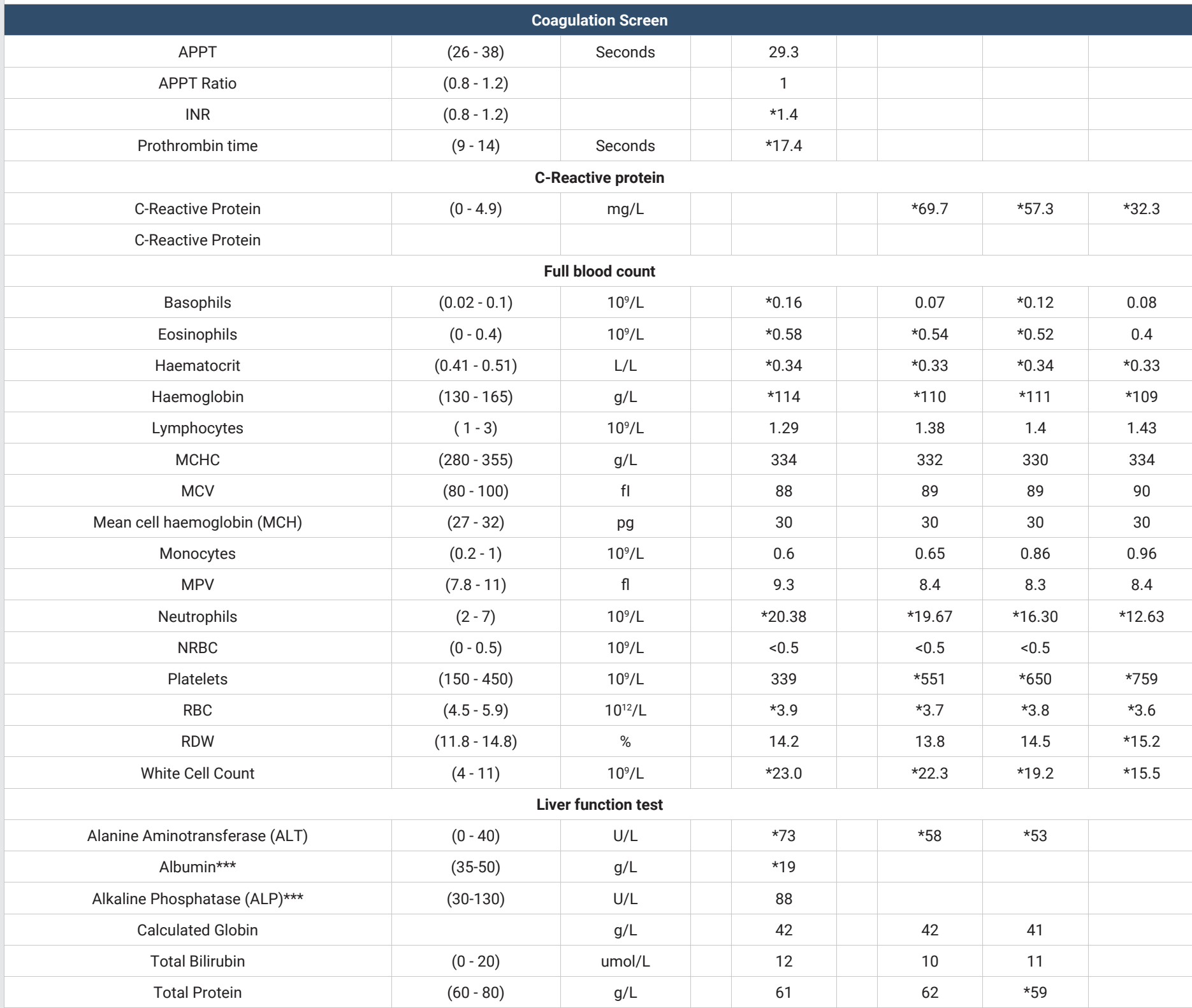

Given his clinical improvement and the normalization of his lab results, the patient was discharged back to the community with a plan to have a cardiac MRI and to follow up in heart failure clinic in 2 to 3 months.

\section{Discussion}

A few case reports of acute heart failure and/or left ventricular dysfunction suggesting myocarditis have been published in the setting of acute SARS-CoV-2 infection. Clinical, laboratory and initial echocardiography findings in this case, support a diagnosis of acute myocarditis. The dynamics of fever, heart rate, increased troponins and CRP seem to best fit a myocardial injury secondary to an inflammatory response. After carefully ruling out usual aetiologies of myocarditis such as common viruses, bacteria, vasculitis and autoimmune disease, we hypothesized that the extrapulmonary manifestations were due to SARS-COV-2 infection. This young patient suffered from a multisystem inflammatory syndrome and acute heart failure with quick recovery in about 10 days suggesting that the myocardial involvement could have been due to myocardial stunning/oedema rather than myocardial necrotic damage $[9,10]$.

This case differs from other COVID-19 patients in some respects. The patient developed acute myocarditis with multiorgan involvement in the absence of obvious preceding respiratory symptoms, with clinical and laboratory evidence of myocarditis improving rapidly. We initially thought food poisoning was the cause of hospitalization due to his initial presentation of fever, diarrhoea and vomiting after eating out at a restaurant. Testing positive for both Covid-19 IgG and IgM likely meant that it was a cause of subacute myocarditis due to SARS-covid-2 virus. Repeat echocardiography testing demonstrated normalized ejection fraction with only residual signs of myocardial dysfunction. This rapid improvement suggests that, in the absence of pre-existing heart conditions, a healthy heart prior to infection among young patients may 
be protective against the higher morbidity and mortality associated with a potential SARS-CoV-2 cardiac involvement [11].

\section{Conclusion}

There is a rising number of covid-19 infection in young patient with extra pulmonary symptoms. Though SARS-CoV-2 can mainly affect lungs, myocardial injury may be frequently evidenced in patients with COVID-19 with variable clinical manifestation. The importance of this case lies in highlighting the severe cardiac involvement in a young patient, without previous risk factors, positive for COVID-19, and the favourable response to supportive care and medical therapy.

\section{Learning points}

- Cardiac and multiorgan involvement may be present in the absence of respiratory symptoms in COVID-19.

- A favourable response to supportive care and medical therapy to severe COVID-19 myocarditis in this young patient with a healthy cardiac system prior to infection was found.

\section{References}

1. Prabhakaran D, Perel P, Roy A, Singh K, Raspail L, et al. (2020) Management of Cardiovascular Disease Patients with Confirmed or Suspected COVID-19 in Limited Resource Settings. Link: https://bit.ly/37ALqbg
2. Pirzada A, Mokhtar A, Moeller A (2020) COVID-19 and Myocarditis: What Do We Know So Far?. CJC Open 2: 278-285. Link: https://bit.ly/3mBax3J

3. Symptoms of Coronavirus and Myocarditis - Myocarditis Foundation. (2020) Link: https://bit.ly/3oicrXr

4. ESC Guidance for the Diagnosis and Management of CV Disease during the COVID-19 Pandemic (2020). Link: https://bit.ly/2JInF8x

5. Tissières $P$, Teboul JL (2020) SARS-CoV-2 post-infective myocarditis: the tip of COVID-19 immune complications? Ann Intensive Care 10: 98. Link: https://bit.ly/2KUjsPO

6. College of Cardiology. (n.d.). Cardiac Sequalae in Severe COVID-19 Patients: NEJM Case Study Instructive. Link: https://bit.ly/2VxbGxA

7. www.jwatch.org. (n.d.). NEJM Journal Watch: Summaries of and commentary on original medical and scientific articles from key medical journals. [online] Link: https://bit.ly/33DKvFP

8. M, Eshak N, Saleh Y, Almaghraby A (2020) Coronavirus Disease 2019 Myocarditis: Insights into Pathophysiology and Management. Journal Coronavirus Disease 2019 Myocarditis: Insights into Pathophysiology and Management. Link: https://bit.ly/37yHp7b

9. Talasaz AH, Kakavand H, Van Tassell B, Aghakouchakzadeh M, Sadeghipour P, et al. (2020) Cardiovascular Complications of COVID-19: Pharmacotherapy Perspective. Cardiovascular Drugs and Therapy. Link: https://bit.ly/2I3vLs0

10. SS, Petraco R, Sen S (2020) Optimal management of acute coronary syndromes in the era of COVID-19. Heart 106. Link: https://bit.ly/3mDsE9a

11. L, Neyts J (2020) Medical treatment options for COVID-19. European Heart Journal: Acute Cardiovascular Care 9: 209-214. Link: https://bit.ly/3qnvZLV
Discover a bigger Impact and Visibility of your article publication with Peertechz Publications

Copyright: (c) 2020 Tun T, et al. This is an open-access article distributed under the terms of the Creative Commons Attribution License, which permits unrestricted use, distribution, and reproduction in any medium, provided the original author and source are credited. 